\title{
Review
}

\section{Biosynthesis and biological function of sulfoglycolipids}

\author{
By Koichi HoNKE*1,†
}

(Communicated by Kunihiko SuZUKI, M.J.A.)

\begin{abstract}
Sulfation confers negative charge on glycolipids and the attached sulfate group presents a part of determinants for the molecular interactions. Mammalian sulfoglycolipids are comprised of two major members, sulfatide ( $\mathrm{SO}_{3}-3 \mathrm{Gal}$-ceramide) and seminolipid $\left(\mathrm{SO}_{3}-3 \mathrm{Gal}-\right.$ alkylacylglycerol). Sulfatide is abundant in the myelin sheath and seminolipid is unique to the spermatogenic cells. The carbohydrate moiety of sulfatide and seminolipid is biosynthesized via sequential reactions catalyzed by common enzymes: ceramide galactosyltransferase (CGT) and cerebroside sulfotransferase (CST). To elucidate the biological function of sulfoglycolipids, we have purified CST, cloned the CST gene, and generated CST-knockout mice. CST-null mice completely lack sulfoglycolipids all over the body. CST-null mice manifest some neurological disorders due to myelin dysfunction, an aberrant enhancement of oligodendrocyte terminal differentiation, and an arrest of spermatogenesis. CST-deficiency ameliorates L-selectin-dependent monocyte infiltration in the renal interstitial inflammation, indicating that sulfatide is an endogenous ligand of L-selectin. Studies on the molecular mechanisms underlying the biological events for which sulfoglycolipids are essential are ongoing.
\end{abstract}

Keywords: cerebroside sulfotransferase (CST), L-selectin, myelin, seminolipid, spermatogenesis, sulfatide

\section{Introduction}

Glycolipids are membrane lipids with a carbohydrate attached. They are located predominantly in the outer leaflet of the plasma membrane and contribute to cell recognition and membrane microdomain formation during various biological events such as differentiation, development and immune reactions. Sulfation, phosphorylation and sialylation confer negative charge on glycolipids and such

*1 Department of Biochemistry and Kochi System Glycobiology Center, Kochi University Medical School, Kochi, Japan.

$\dagger$ Correspondence should be addressed: K. Honke, Department of Biochemistry, Kochi University Medical School, Kohasu, Oko-cho, Nankoku, Kochi 783-8505, Japan (e-mail: khonke@kochiu.ac.jp).

Abbreviations: BORIS: brother of the regulator of imprinted sites; CGT: ceramide galactosyltransferase; CST: cerebroside sulfotransferase; GalCer: galactosylceramide; GalEAG: galactosylalkylacylglycerol; Gal3ST: $\beta \mathrm{Gal} 3$ - $O$-sulfotransferase; MCT: monocarboxylate transporter; MRI: magnetic resonance imaging; PAPS: 3'-phosphoadenosine 5'-phosphosulfate; PPAR: peroxisome proliferator-activated receptor; UTR: untranslated region; UUO: unilateral ureteral obstruction. structures form a part of determinants for the molecular interactions.

The first one of the mammalian sulfoglycolipids, sulfatide was isolated from the human brain by Thudichum in as early as 1884 (reviewed in Ref. 1). The correct structure of sulfatide was determined by Yamakawa in 1962..2) Thereafter, many kinds of sulfoglycolipids have been discovered from various organs. ${ }^{1)}$ Among them is a unique testis-specific seminolipid..$^{3), 4)}$ The carbohydrate moiety of sulfatide and seminolipid has the same structure, $\mathrm{HSO}_{3}-3 \mathrm{Gal}$ but their lipid moiety is different from each other (Fig. 1). Sulfatide is a sphingolipid, while seminolipid is a glycerolipid. The distribution of sulfoglycolipids is tissue-specific and they are abundant in myelin, spermatozoa, kidney, and alimentary canal. ${ }^{1)}$ Especially, sulfatide is a major lipid component of the myelin sheath and synthesized in myelin-generating cells, oligodendrocytes in the central nervous system and Schwann cells in the peripheral nervous system. ${ }^{1,5)}$ On the other hand, seminolipid is synthesized in spermatocytes and maintained in the subsequent germ cell stages. ${ }^{1,5)}$ The carbohydrate 


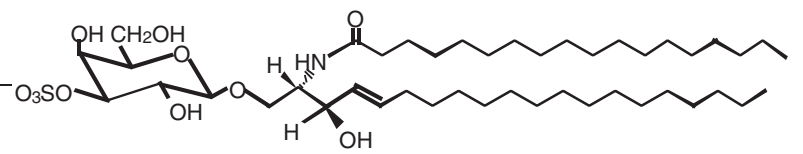

Sulfatide

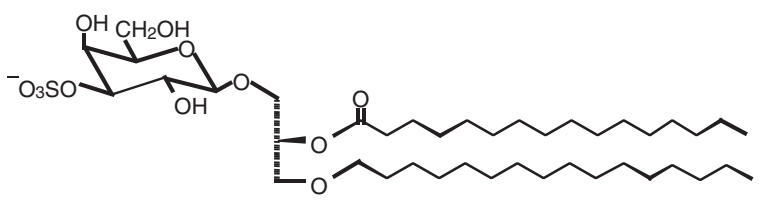

\section{Seminolipid}

Fig. 1. Structure of sulfatide and seminolipid.

\section{Ceramide (Cer) $\downarrow \underset{\text { (CGT) }}{\text { ceramide galactosyltransferase }} \downarrow$ \\ GalCer \\ GalEAG \\ Sulfatide \\ Seminolipid}

Fig. 2. Biosythetic pathway of sulfatide and seminolipid.

moiety of sulfatide and seminolipid is biosynthesized via sequential reactions catalyzed by common enzymes: ceramide galactosyltransferase (CGT, EC 2.4.1.45) and cerebroside sulfotransferase (CST, EC 2.8.2.11) (Fig. 2). Sulfoglycolipids have been implicated in a variety of physiological functions through their interactions with extracellular matrix proteins, cellular adhesive receptors, blood coagulation systems, and microorganisms. ${ }^{1,5)}$

\section{Purification and molecular cloning of human CST}

Since glycolipid sulfotransferase was identified as an enzyme to catalyze the transfer of a sulfate group from the sulfate donor, PAPS to the C3 position of the Gal residue of galactosylceramide (GalCer) ${ }^{6)}$ it was originally called cerebroside sulfotransferase (cerebroside is the trivial name of GalCer), and therefore abbreviated CST. Later, CST has been demonstrated to act not only on GalCer but also on galactosylglycerolipids, lactosylceramide,

glucosylceramide, gangliotriaosylceramide, and gangliotetraosylceramide, ${ }^{7)-9)}$ indicating that CST prefers $\beta$-glycoside, especially $\beta$-galactoside, at the nonreducing termini of sugar chains attached to a lipid moiety.

1-1. Purification of CST from human renal cancer cells. Kidney is one of the tissues rich in sulfoglycolipids and hence has been employed to study their biosynthesis. ${ }^{7), 8)}$ Makita and colleagues have demonstrated that human renal cell carcinoma tissue $^{10)}$ and a cell line derived therefrom, ${ }^{11)}$ accumulate sulfoglycolipids secondary to a marked elevation of CST activity, and that CST activity in renal cancer cells is enhanced by the actions of growth factors such as epidermal growth factor (EGF), ${ }^{12)}$ transforming growth factor- $\alpha(\mathrm{TGF}-\alpha)^{13)}$ and hepatocyte growth factor (HGF). ${ }^{14)}$ Furthermore, tyrosine-kinase, ${ }^{15)}$ protein kinase-C, ${ }^{16)}$ and $\operatorname{Ras}^{17)}$ have been shown to be involved in the expression of CST in renal cancer cells.

Since the CST gene had yet to be cloned at that time, we undertook to purify the enzyme from human renal cancer cells and eventually succeeded in purification through a combination of affinity chromatographies using galactosylsphingosine (an acceptor analog), 3',5'-bisphosphoadenosine (a donor analog), and heparin as ligands. ${ }^{9)}$ The purified CST showed a specific activity of $1.2 \mu \mathrm{mol} / \mathrm{min} / \mathrm{mg}$, which was 300 times more than the highest activity among the enzyme preparation purified so far from other sources. ${ }^{8), 18), 19)}$ Homogeneity of the purified CST was supported by the findings that the enzyme preparation showed a single protein band with an apparent molecular mass of $54 \mathrm{kDa}$ on reducing SDSPAGE, and that protein bands coincided with the enzyme activity on both native PAGE and nonreducing SDS-PAGE. ${ }^{9)}$

1-2. cDNA and genomic DNA cloning of human CST. We have cloned a cDNA encoding the human $\mathrm{CST}^{20)}$ on the basis of the partial amino acid sequence of the purified enzyme. ${ }^{9)}$ The isolated cDNA clone contained an open reading frame encoding 423 amino acids. The deduced amino acid sequence predicts a type II transmembrane topology and contains two potential $N$-glycosylation sites and putative PAPS binding sites. $^{21)}$ Then we have determined the genomic organization of the human CST gene. ${ }^{22)}$ The gene comprises at least four exons and spans about $20 \mathrm{~kb}$. The coding region is located in exons 3 and 4 . We found four transcription initiation sites and alternative usage of six exons (exons $1 \mathrm{a}-\mathrm{d}$ and $2 \mathrm{a}-\mathrm{b}$ ) corresponding to the $5^{\prime}$-UTR 
in renal cancer cells, while the only transcript beginning at the exon 1a was observed in normal cells. All the mRNA variants with different 5 '-UTRs were found to produce the identical proteins, suggesting that aberrant usage of transcription initiation sites flanked with promoters/enhancers is involved in the cancer-associated overexpression of the CST gene. ${ }^{23)}$ Furthermore, the human CST gene was assigned to the chromosome $22 \mathrm{q} 12$ by means of fluorescence in situ hybridization. ${ }^{22}$

When the CST gene was cloned, it showed no homologous genes, suggesting that CST has a unique evolutional origin. ${ }^{20}$ Thereafter the second $\beta \mathrm{Gal} 3-O$ sulfotransferase (Gal3ST) termed GP3ST that acts on Gal $\beta 1-3 / 4 \mathrm{GlcNAc-R}$ oligosaccharides was identified based on its similarity to the CST gene. ${ }^{24)}$ This finding indicated the existence of Gal3ST gene family. Subsequently, two other members of this family have been identified ${ }^{25)-27)}$ and the terminology of Gal3ST-1 through 4 was proposed. ${ }^{26)}$ Gal3ST-3 exclusively acts on type 2 chain in $N$ - and $O$ glycans $^{25), 26)}$ but also acts on Gal $\beta 1-4$ (sulfo6)GlcNAc-R oligosaccharide. ${ }^{26)}$ Gal3ST-4 recognizes Gal $\beta 1-3$ GalNAc-R and Gal $\beta 1-3($ GlcNAc $\beta 1-6)$ GalNAc-R as good acceptors but not Gal $\beta 1-3 /$ 4GlcNAc-R. ${ }^{27)}$ Neither Gal3ST-2, Gal3ST-3 nor Gal3ST-4 acted on GalCer ${ }^{24)-27)}$ in good agreement with the finding obtained by the CST-knockout mice that the single enzyme is responsible for the biosynthesis of sulfoglycolipids. ${ }^{28)}$

\section{Expression and gene targeting of the mouse Cst gene}

Tissue-specific carbohydrate structures are considered to result from the selective expression of specific glycosyltranferases and carbohydrate-modifying enzymes such as sulfotransferases responsible for their synthesis. CST activity has been demonstrated in brain, testis, kidney, gastric mucosa, lung and endometrium of various mammals, ${ }^{1)}$ i.e. tissues in which sulfoglycolipids are expressed. To elucidate the regulatory mechanism of tissue-specific expression of sulfoglycolipids, we investigated the organization of the mouse Cst gene. $^{29)}$

2-1. Tissue-specific expression system of mouse CST. First, we isolated a mouse cDNA clone encoding CST from a kidney cDNA library, using a human CST cDNA clone ${ }^{20}$ as a probe. A recombinant protein of the cloned cDNA showed CST activity. The deduced protein is composed of the same 423 amino acids as human CST and its sequence exhibits $84 \%$ identity with that of the human ortholog. The mouse Cst gene is preferentially transcribed in stomach, small intestine, brain, kidney, lung and testis, in that order. When we analyzed the sequence of transcripts in various tissues, we found seven different nucleotide sequences in the $5^{\prime}$-UTR, while the sequence in the coding region was identical in all the transcripts.

Next, we isolated Cst genomic DNA clones from a mouse genomic library. ${ }^{29)}$ The clones covered all the 5'-UTR sequences and coding exons including 3 'UTR. RT-PCR analyses of Cst mRNAs from various tissues confirmed that Cst transcripts are tissuespecifically spliced by alternative use of multiple exons 1 (exons $1 \mathrm{a}-\mathrm{g}$ ). These observations suggest that the tissue-specific expression of the murine Cst gene is explained by alternative usage of multiple $5^{\prime}$ UTR exons flanked with tissue-specific promoters. ${ }^{29)}$ For example, transcription of the Cst gene in the testis is initiated at exon $1 \mathrm{f}$ and activated by a CTCF paralogous transcriptional factor, brother of the regulator of imprinted sites (BORIS). ${ }^{30)}$ The BORIS target site was identified in the $5^{\prime}$-flanking region of exon 1f. By contrast, peroxisome proliferator-activated receptor $\alpha(\operatorname{PPAR} \alpha)$ activates the transcription of the Cst gene in the kidney, heart, liver, and small intestine. ${ }^{31)}$ The PPAR response element is found in the DNA sequences upstream of exon 1a and exon 1f. However, it remains to be solved whether these elements are actually responsible for the expression of CST.

2-2. Generation of CST-knockout mice. Insight into the physiological function of sulfoglycolipids was provided by gene-targeted disruption of CGT. ${ }^{32), 33)}$ This single enzyme, CGT, is responsible for the biosynthesis of GalCer and GalEAG, which are the precursors of sulfatide and seminolipid, respectively (Fig. 2). CGT-knockout mice are completely devoid of GalCer and sulfatide in brain ${ }^{32), 33)}$ and of GalEAG and seminolipid in testis. ${ }^{34)}$ CGTdeficient mice manifest neurological disorders caused by myelin dysfunction ${ }^{32), 33)}$ and the complete inhibition of spermatogenesis at the late-pachytene spermatocyte stage. ${ }^{34)}$ These results indicated that the intermediate products, GalCer and GalEAG, and/or the end products, sulfatide and seminolipid, are indispensable for myelin function and spermatogenesis. However, generation of CST-deficient mice was required to distinguish the function of GalCer and GalEAG from that of sulfatide and seminolipid.

To address this problem and to determine whether a single enzyme is responsible for the biosynthesis of both sulfatide and seminolipid in vivo, 


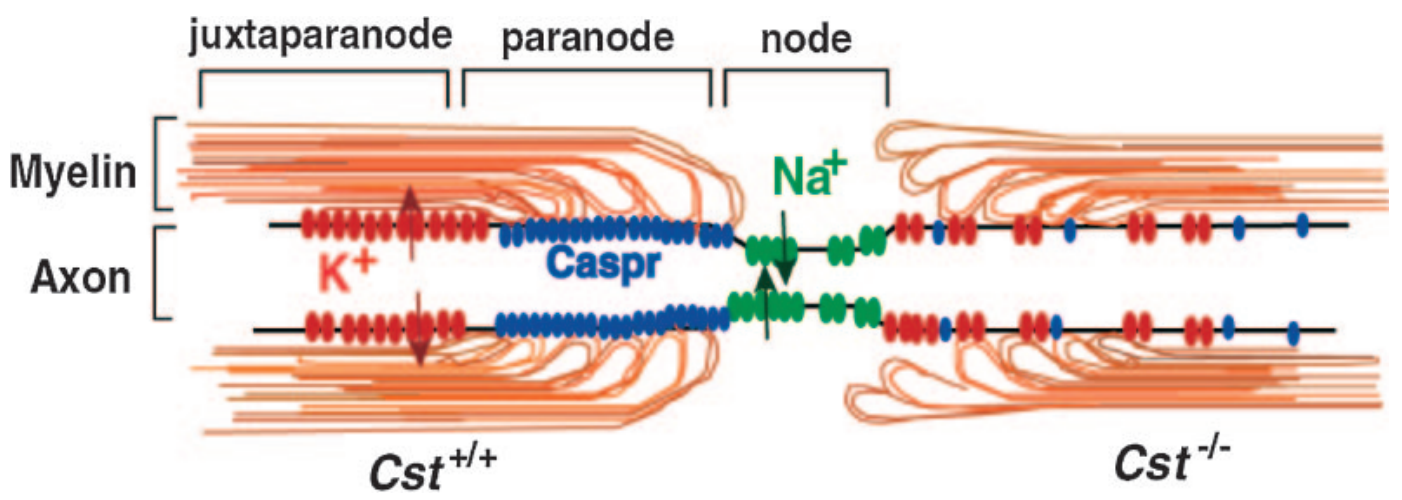

Fig. 3. Organization of nodes of Ranvier and its alterations in CST-deficient mice. In wild-type mice (left side), myelin membranes and axon membranes form junctions at the paranode region and $\mathrm{Na}^{+}$and $\mathrm{K}^{+}$channels cluster at the node and juxtaparanode regions, respectively, separated by the septate-like axo-glial junction. In CST-deficient mice (right side), formation of the axo-glial junction is disordered and the clustering of $\mathrm{Na}^{+}$and $\mathrm{K}^{+}$channels is also disturbed.

CST-knockout mice were generated by means of gene targeting in our lab. ${ }^{28)}$ CST activity disappears in the whole body of $C s t$-null mice. The mutant mice show a complete loss of sulfatide in brain and seminolipid in testis, proving that a single gene copy is responsible for the biosynthesis of both sulfatide and seminolipid. CST-knockout mice demonstrated the essentiality of sulfoglycolipids in myelin function and spermatogenesis as described below.

2-3. Sulfatide in myelin function and oligodendrocyte differentiation. Mammals have acquired property known as myelin during the evolution so as to enhance the conductivity of the neuronal impulse. Oligodendrocytes produce vast amounts of myelin, a unique and lipid-rich biomembranes with a relatively simple array of myelinspecific proteins in the central nervous system. This membrane, an extension of the oligodendrocyte plasma membrane, forms multilamellar and spirally wrapped sheaths around neuronal axons. The gaps between adjacent myelin sheaths are referred to as nodes of Ranvier, and myelin forms lateral loops there (reviewed in Ref. 35) (Fig. 3). These myelin loops terminate at the paranode region and engage in the formation of a septate-like adhesive junction with the axon membrane, axolemma. This specialized axoglial junction acts as an electronical and biochemical barrier between nodal and internodal membrane compartments. Voltage-gated sodium channels concentrate in the nodal axolemma, while shaker-type $\mathrm{K}^{+}$channels, Kv1.1 and Kv1.2, localize within the juxtaparanodal axolemma. Saltatory conduction of the action potential is attributed to this organization. The adhesion of myelin to the axolemma plays a critical role in this clustering of ion channels. Thus myelin serves not only as a simple insulator but also as a functional platform of the neuron-glia interaction.

The remarkable biochemical characteristics that distinguishes myelin from other membranes are its high lipid content accounting for more than $70 \%$ of the dry weight of myelin membranes and lipid composition. ${ }^{1)}$ The myelin membrane contains a high level of cholesterol and galactosphingolipids. GalCer and sulfatide comprise $23 \%$ and $4 \%$ of the total lipid content in the myelin sheath, respectively. ${ }^{1)}$ In attempts to better understand myelin function, a number of mouse models modifying lipid metabolism have been investigated (reviewed in Ref. 36). As described above, the first animal studies to investigate the role of sulfatide in the myelin function have been performed using CGT-knockout mice. ${ }^{32), 33)}$ Since CGT-deficient mice lack GalCer as well as sulfatide, and exhibit other secondary changes in the lipid composition of myelin, including accumulation of 2-hydroxy glucosylceramide and sphingomyelin, it was impossible to segregate the specific function of GalCer, sulfatide and the other changes. To solve this problem, we generated CST-knockout mice. ${ }^{28)}$ As expected, CST-deficient mice completely lack sulfatide, whereas other glycolipids including GalCer are not significantly altered in brain.

CST-null mice were born healthy, but began to display hindlimb weakness by 6 weeks of age and subsequently showed a pronounced tremor and progressive ataxia. ${ }^{28)}$ Histological analysis revealed that axons were well myelinated in CST-null mice. Electron microscopic analysis of myelinated nerve fibers, however, revealed disorganized termination of the lateral loops at the node of Ranvier, similar 
to that reported in CGT-deficient mice ${ }^{37)}$ (Fig. 3). Furthermore, clustering of $\mathrm{Na}^{+}$and $\mathrm{K}^{+}$channels at the node is also deteriorated in CST-null mice ${ }^{38)}$ as observed in CGT-null mice ${ }^{39)}$ (Fig. 3). These paranodal junction abnormalities are observed by magnetic resonance imaging (MRI), in which the $\mathrm{T} 1$ time is lower and the T2 time is higher. ${ }^{40)}$ Moreover, diffusion tensor MRI (DTI) shows lower axial diffusivity and higher radical diffusivity. ${ }^{40)}$ Thus sulfatide is an indispensable molecule for the axoglial junction between myelin loop and axolemma at the paranode region. The $155 \mathrm{kDa}$ splice isoform of neurofascin (NF155) located on the myelin membrane and Caspr/paranodin and contactin that are on the axolemma collaborate to form the axo-glial adhesion apparatus. ${ }^{35)}$ It is an open question how sulfatide interacts with these protein complexes and maintain the adhesive junction.

Despite the significant neurological disorders, CST-null mice are able to survive to more than one year of age. ${ }^{28)}$ The phenotype of the CST-deficient mice was milder than that of CGT-deficient mice $^{32), 33)}$ in terms of the age of onset, life span, and the severity of symptoms. The most striking difference between these two mutants is the reduction of myelin developmental abnormalities in young adult CST-null mice as compared with CGT-null mice. ${ }^{41)}$ Sulfatide is needed for myelin maintenance rather than myelin development, because the prevalence of redundant, uncompacted, and degenerating myelin sheaths as well as deteriorating nodal/paranodal structure is increased with age. ${ }^{41)}$ The discrepancy in phenotype between CST- and CGT-null mice suggests that GalCer not only acts as a precursor for sulfatide synthesis, but also serves as a particular function.

Since sulfatide emerges when oligodendrocyte progenitors cease dividing and commence differentiating, it is supposed to be involved in the regulation of terminal differentiation. In fact, this terminal differentiation is enhanced in CGT-KO mice. ${ }^{42)}$ Furthermore, oligodendrocyte differentiation in wild-type mice was found to be blocked by antisulfatide antibody but not by anti-GalCer antibody. ${ }^{43)}$ These findings strongly suggest that sulfatide is a key negative regulator of the oligodendrocyte differentiation. This hypothesis was certified by the fact that terminal differentiation and morphological maturation of oligodendrocytes are enhanced in cultures of CST-deficient mice. ${ }^{44)}$ Furthermore, elevated numbers of differentiated oligodendrocytes were observed in the spinal cord, brain and optic nerves of CST-null mice. ${ }^{44-46)}$ Hence sulfatide plays a critical role in the regulation of oligodendrocyte terminal differentiation, in addition to their eventual roles as structural components of mature myelin.

2-4. Seminolipid in spermatogenesis. Mammalian spermatogenesis is a complex, highly organized process that takes place in the seminiferous tubules of the testis, in which germ cells undergo proliferation and differentiation to become spermatozoa (reviewed in Ref. 47). The proliferation and differentiation of spermatogonial stem cells occur in the basal compartment at the peripheral side of the seminiferous tubules. These differentiated spermatogonia then become spermatocytes and begin meiosis, migrating from the peripheral to the luminal side along somatic Sertoli cells. The mutual interaction between germ cells and Sertoli cells play a crucial role in their differentiation (for a review, see Ref. 48). After spermatogenesis in the testis, spermatozoa are released into the lumen of seminiferous tubules and are transported to the epididymis where they continue to mature.

More than $90 \%$ of glycolipid in the testis consists of a unique glyceroglycolipid, seminolipid. ${ }^{1), 5)}$ Its carbohydrate moiety is identical to that of sulfatide (Fig. 1) and the synthesizing enzymes are common (Fig. 2). Disruption of gene for these enzymes in mice results in the complete absence of seminolipid in the testis and male infertility due to the arrest of spermatogenesis. ${ }^{28), 34)}$ The primary spermatocytes seem to be normal in CST-deficient mice, but spermatogenesis is blocked at the metaphase of the first meiosis. ${ }^{28)}$ The arrested stage in the germ cell differentiation of CST-null mice appears to be somewhat later than that in CGT-null mice, ${ }^{34)}$ suggesting that both GalEAG and seminolipid are successively involved in the genetic program of spermatogenesis in the same order as their biosynthesis.

Although these glycolipids were reported to express on the cell surface of primary spermatocytes from the end of the leptotene stage or the zygotene stage and later, ${ }^{1,5)}$ the issue of which side of cell function, germ cells or Sertoli cells, is deteriorated in these mutant mice remained unknown. To address this question, we examined whether spermatogenesis is restored or not after testis germ-cell transplantation, ${ }^{49)}$ in which wild-type spermatogonial stem cells were injected into the seminiferous tubules of CSTnull mice. As the result, the transplanted green fluorescent protein-positive cells generated colonies and spermatogenesis proceeded over meiosis in the mutant testis, ${ }^{50)}$ indicating that the defect is in the 


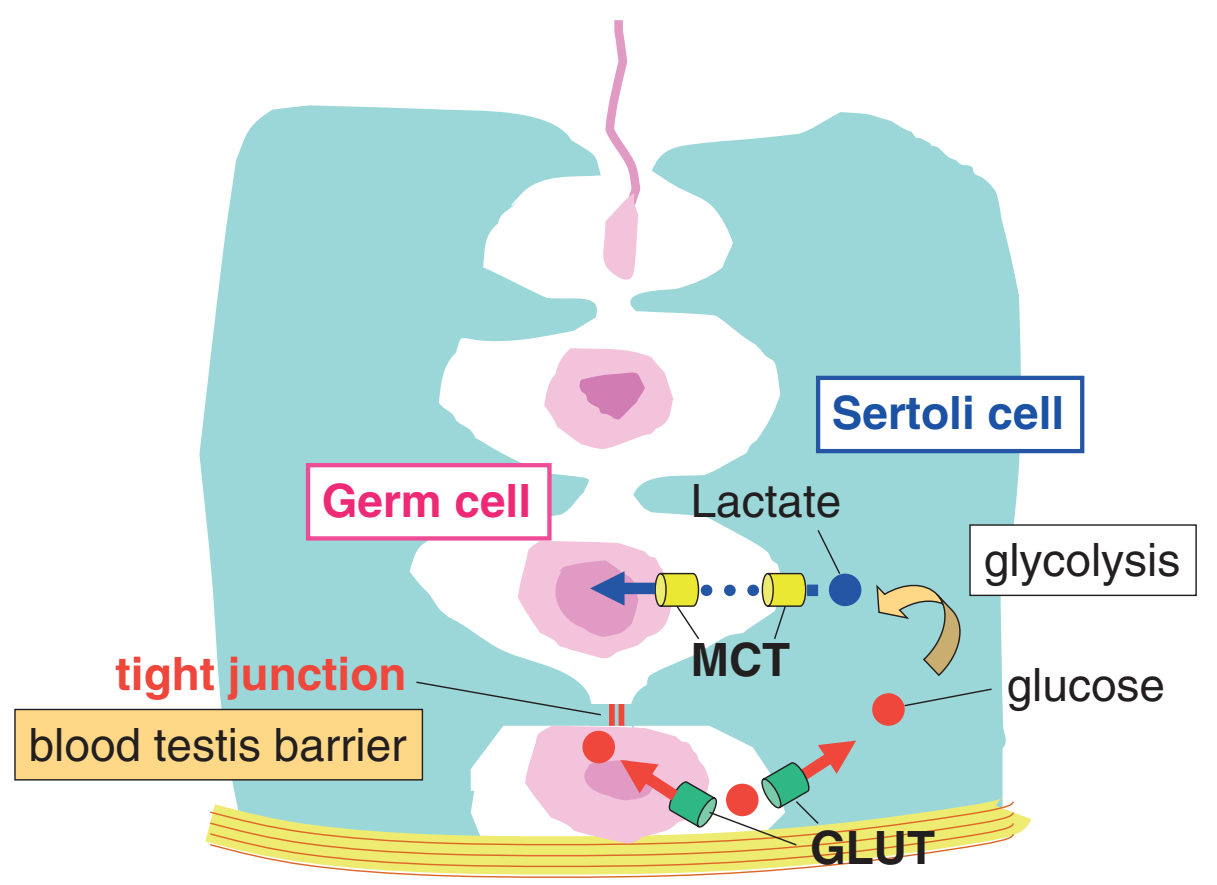

Fig. 4. Lactate is the main energy source for male germ cells. The basal side of the blood testis barrier (interstitial compartment) is connected to blood and contains glucose, but the apical side (adluminal compartment) lacks glucose. Energy fuel is obtained in a form of lactate that is made from glucose in Sertoli cell. The release and uptake of lactate are mediated by monocarboxylate transporters (MCTs). GLUT, glucose transporter.

germ cell side. The immunostaining of wild-type testes using an anti-sulfoglycolipid monoclonal antibody in comparison with $C$ st-null testes as a negative control demonstrated that seminolipid is expressed on the plasma membranes of Type B spermatogonia, spermatocytes, spermatids, and spermatozoa. ${ }^{50)}$

Energy metabolism in the testes exhibits some specificity in that lactate is the central energy metabolite used by germ cells (reviewed in Ref. 51). Spermatocytes are located inside the blood testis barrier (adluminal compartment), whereas spermatogonia are outside the barrier (interstitial compartment). Because glucose is unable to pass through the barrier, spermatocytes cannot utilize glucose derived from the blood as an energy source (Fig. 4). Glucose is taken up and converted to lactate by Sertoli cells. Then lactate is transported to and used by germ cells to synthesize ATP. Our recent study revealed that the Cst-null spermatocytes are not able to take up lactate, resulting in ATP depletion and apoptosis (unpublished result by Yamashita and Honke). We hypothesize that seminolipid is involved in the intracellular traffic of a lactate transporter (MCT) in spermatocytes.

2-5. Other functions of sulfatide. In the experimental model of kidney interstitial inflamma- tion, unilateral ureteral obstruction (UUO) leads to infiltration of monocytes into interstitium. Monocyte infiltration is a multistep process in which chemokine and adhesion molecules play key roles. The initial step of this process involves the binding of monocytes to the endothelium of venules, mediated by various adhesion molecules such as selectins. The monocyte infiltration following UUO is inhibited by a neutralizing antibody against L-selectin. ${ }^{52)}$ The main Lselectin ligand activity in lymphoid organs is served by sialylated, fucosylated, and sulfated glycans on mucin-like molecules, but it also binds to sulfatide. ${ }^{53)}$ Since distribution of sulfatide, which is demonstrated by anti-sulfatide monoclonal antibody, was similar to that of L-selectin ligand and exogenously added sulfatide considerably inhibited the monocyte infiltration after UUO treatment, ${ }^{52)}$ sulfatide was supposed to be involved in the monocyte infiltration mediated by L-selectin. This hypothesis was tested using CST-null mice. ${ }^{54)}$ The distributions of Lselectin ligand activity and sulfatide were relocated from the distal tubules to the endothelium of peritubular capillaries, where monocytes infiltrate, after UUO treatment in wild-type mice. In contrast, the L-selectin ligand activity was not detected in CST-null mice irrespective of UUO treatment. 
Compared with wild-type mice, CST-null mice showed a considerable reduction in the number of monocytes/macrophages that infiltrate into the interstitium after UUO to a similar extent in Lselectin-null mice. These findings indicate that sulfatide is a major L-selectin ligand in the kidney and that the interaction between L-selectin and sulfatide plays a central role in monocyte infiltration into the kidney interstitium.

Sulfatide interacts with a variety of cell adhesion molecules including fibrinogen, von Willebrand factor (VWF), P-selectin, thrombospondin and laminin, ${ }^{1,5)}$ which are involved in hemostasis. A sulfatide-specific single-chain fragment variable $(\mathrm{scFv})$ antibody PA38 inhibits the adhesion of activated platelets to fibrinogen, VWF, P-selectin, TSP1 and laminin. Furthermore, PA38 inhibits the collagen, ADP, thrombin and ristocetin-induced platelet aggregation in isolated platelet-rich plasma. The collagen-induced platelet aggregation is suppressed in Cst-null mice, although in vivo hemostasis is preserved. ${ }^{55)}$ Enhanced adhesion of sickle erythrocytes to the vascular endothelium and subendothelial matrix is fundamental to the development of vascular occlusion in sickle cell disease. The previous evidence linking sulfatide to cell adhesion implied that erythrocyte membrane sulfatide is involved in the vasoocclusive crises in sickle cell disease patients. The adhesion of sickle erythrocytes to endothelium is prevented by PA38 and the extent of adhesion of Cst-null erythrocytes is reduced, suggesting a role for sulfatide in sickle cell disease pathophysiology. ${ }^{56}$ ) Taken together with these minor effects, sulfoglycolipids might be involved in a broad range of biological events all over the body.

\section{Perspectives}

Our studies on the biosynthesis and the biological function of sulfoglycolipids have revealed that sulfoglycolipids are indispensable for organisms. Now we have to elucidate molecular mechanisms how sulfoglycolipids work in the biological processes in which sulfoglycolipids are proved to be essential. Since sulfoglycolipids are small molecules and occupy only in the outer leaflet of the plasma membrane, it is assumed that they need to collaborate with other functional molecules. For instance, sulfatide is supposed to associate with the adhesive proteins in the paranodal axo-glial junction. Sulfatide may directly interact with molecules on the opposite cells, which is true for the L-selectin-sulfatide interaction mediating the monocyte filtration in the kidney.
Alternatively, sulfatide may interact with other molecules located on the same membrane plane. In general, glycosphingolipids self-associate in cellular membranes to form a microdomain referred to lipid rafts. Glycosylphosphatidylinositol-anchored proteins, which contain ether glycerolipid like seminolipid, are also enriched in this microdomain. The fact that sulfatide and seminolipid are recovered in the detergent-insoluble floating membrane fractions suggests that sulfoglycolipids are included in these membrane microdomains. These microdomains are proposed to serve as platforms for receptor signaling, cell adhesion and intracellular protein trafficking. To elucidate the functional molecules that collaborate with sulfoglycolipids and the role of sulfoglycolipids in the organization of such functional platforms is an inevitable challenge in the future.

Sulfatide is a multifunctional glycolipid. Some bacteria and viruses utilize sulfatide for processes of their infection and replication. ${ }^{57)}$ For instance, the association of sulfatide with hemagglutinin delivered to the cell surface is found to enhance the replication of influenza A virus (IAV) by promoting translocation of the newly synthesized IAV ribonucleoprotein complexes from the nucleus to the cytoplasm. ${ }^{58)}$ Elucidation of the detailed molecular mechanism of this process might provide a clue for development of an innovative antiviral drugs.

\section{Acknowledgements}

The author thanks all the collaborators concerned with the studies described in this review article. This study was supported by Grant-in-Aid for Scientific Research from the Ministry of Education, Culture, Sports, Science and Technology of Japan and Core Research for Evolutional Science and Technology (CREST), Japan Science and Technology Agency.

\section{References}

1) Ishizuka, I. (1997) Chemistry and functional distribution of sulfoglycolipids. Prog. Lipid Res. 36, 245-319.

2) Yamakawa, T., Kiso, N., Handa, S., Makita, A. and Yokoyama, S. (1962) On the structure of brain cerebroside sulfuric ester and ceramide dihexoside of erythrocytes. J. Biochem. 52, 226-227.

3) Ishizuka, I., Suzuki, M. and Yamakawa, T. (1973) Isolation and characterization of a novel sulfoglycolipid, 'seminolipid,' from boar testis and spermatozoa. J. Biochem. 73, 77-87.

4) Kornblatt, M.J., Knapp, A., Levine, M., Schachter, H. and Murray, R.K. (1974) Studies on the structure and formation during spermatogenesis 
of the sulfoglycerogalactolipid of rat testis. Can. J. Biochem. 52, 689-697.

5) Vos, J.P., Lopes-Cardozo, M. and Gadella, B.M. (1994) Metabolic and functional aspects of sulfogalactolipid. Biochim. Biophys. Acta 1211, 125149.

6) Farooqui, A.A., Rebel, G. and Mandel, P. (1977) Sulphatide metabolism in brain. Life Sci. 20, 569583.

7) Lingwood, C.A. (1985) Developmental regulation of galactoglycerolipid and galactosphingolipid sulphation during mammalian spermatogenesis. Biochem. J. 231, 393-400.

8) Tennekoon, G., Aitchison, S. and Zaruba, M. (1985) Purification and characterization of galactocerebroside sulfotransferase from rat kidney. Arch. Biochem. Biophys. 240, 932-944.

9) Honke, K., Yamane, M., Ishii, A., Kobayashi, T. and Makita, A. (1996) Purification and characterization of 3 '-phosphoadenosine-5'-phosphosulfate: GalCer sulfotransferase from human renal cancer cells. J. Biochem. 119, 421-427.

10) Sakakibara, N., Gasa, S., Kamio, K., Makita, A. and Koyanagi, T. (1989) Association of elevated sulfatides and sulfotransferase activities with human renal cell carcinoma. Cancer Res. 49, 335-339.

11) Kobayashi, T., Honke, K., Kamio, K., Sakakibara, N., Gasa, S., Miyao, N., Tsukamoto, T., Ishizuka, I., Miyazaki, T. and Makita, A. (1993) Sulfolipids and glycolipid sulfotransferase activities in human renal cell carcinoma cells. Br. J. Cancer 67, 76-80.

12) Kobayashi, T., Honke, K., Gasa, S., Kato, N., Miyazaki, T. and Makita, A. (1993) Epidermal growth factor elevates the activity levels of glycolipid sulfotransferases in renal-cell-carcinoma cells. Int. J. Cancer 55, 448-452.

13) Kobayashi, T., Honke, K., Gasa, S., Imai, S., Tanaka, J., Miyazaki, T. and Makita, A. (1993) Regulation of activity levels of glycolipid sulfotransferases by transforming growth factor $\alpha$ in renal cell carcinoma cells. Cancer Res. 53, 56385642 .

14) Kobayashi, T., Honke, K., Gasa, S., Miyazaki, T., Tajima, H., Matsumoto, K., Nakamura, T. and Makita, A. (1994) Hepatocyte growth factor elevates the activity levels of glycolipid sulfotransferases in renal cell carcinoma cells. Eur. J. Biochem. 219, 407-413.

15) Balbaa, M., Honke, K. and Makita, A. (1996) Regulation of glycolipid sulfotransferase by tyrosine kinases in human renal cancer cells. Biochim. Biophys. Acta 1299, 141-145.

16) Kobayashi, T., Honke, K., Gasa, S., Sugiura, M., Miyazaki, T., Ishizuka, I. and Makita, A. (1993) Involvement of protein kinase $\mathrm{C}$ in the regulation of glycolipid sulfotransferase activity levels in renal cell carcinoma cells. Cancer Res. 53, 2484-2489.

17) Yabunaka, N., Honke, K., Ishii, A., Ogiso, Y., Kuzumaki, N., Agishi, Y. and Makita, A. (1997) Involvement of Ras in the expression of glycolipid sulfotransferase in human renal cancer cells. Int. J.
Cancer 71, 620-623.

18) Sakac, D., Zachos, M. and Lingwood, C.A. (1992) Purification of the testicular galactolipid: $3^{\prime}-$ phosphoadenosine 5'-phosphosulfate sulfotransferase. J. Biol. Chem. 267, 1655-1659.

19) Sundaram, K.S. and Lev, M. (1992) Purification and activation of brain sulfotransferase. J. Biol. Chem. 267, 24041-24044.

20) Honke, K., Tsuda, M., Hirahara, Y., Ishii, A., Makita, A. and Wada, Y. (1997) Molecular cloning and expression of cDNA encoding human $33^{\prime}$ phosphoadenylylsulfate:galactosylceramide 3 '-sulfotransferase. J. Biol. Chem. 272, 4864-4868.

21) Kakuta, Y., Pedersen, L.G., Pedersen, L.C. and Negishi, M. (1998) Conserved structural motifs in the sulfotransferase family. Trends Biochem. Sci. 23, 129-130.

22) Tsuda, M., Egashira, M., Niikawa, N., Wada, Y. and Honke, K. (2000) Cancer-associated alternative usage of multiple promoters of human GalCer sulfotransferase gene. Eur. J. Biochem. 267, 26722679 .

23) Honke, K., Tsuda, M., Hirahara, Y., Miyao, N., Tsukamoto, T., Satoh, M. and Wada, Y. (1998) Cancer-associated expression of glycolipid sulfotransferase gene in human renal cell carcinoma cells. Cancer Res. 58, 3800-3805.

24) Honke, K., Tsuda, M., Koyota, S., Wada, Y., Tanaka, N., Ishizuka, I., Nakayama, J. and Taniguchi, N. (2001) Molecular cloning and characterization of a human $\beta$-Gal 3 '-sulfotransferase which acts on both type 1 and type $2(\mathrm{Gal} \beta 1,3 /$ 1,4GlcNAc-R) oligosaccharides. J. Biol. Chem. 276, 267-274.

25) El-Fasakhany, F.M., Uchimura, K., Kannagi, R. and Muramatsu, T. (2001) A novel human Gal-3-Osulfotransferase: molecular cloning, characterization and its implications in biosynthesis of ( $\left.\mathrm{SO}_{4}-3\right) \mathrm{Gal} \beta 1-4$ (Fuc $\left.\alpha 1-3\right)$ GlcNAc. J. Biol. Chem. 276, 26988-26994.

26) Suzuki, A., Hiraoka, N., Suzuki, M., Angata, K., Misra, A.K., McAuliffe, J., Hindsgaul, O. and Fukuda, M. (2001) Molecular cloning and expression of a novel human $\beta$-Gal-3- $O$-sulfotransferase that acts preferentially on $\mathrm{N}$-acetyllactosamine in $N$ - and O-glycans. J. Biol. Chem. 276, 2438824395

27) Seko, A., Hara-Kuge, S. and Yamashita, K. (2001) Molecular cloning and characterization of a novel human galactose 3 - $O$-sulfotransferase that transfers sulfate to Gal $\beta 1-3 \mathrm{GalNAc}$ residue in $O$ glycans. J. Biol. Chem. 276, 25697-25704.

28) Honke, K., Hirahara, Y., Dupree, J., Suzuki, K., Popko, B., Fukushima, K., Fukushima, J., Nagasawa, T., Yoshida, N., Wada, Y. and Taniguchi, N. (2002) Paranodal junction formation and spermatogenesis require sulfoglycolipids. Proc. Natl. Acad. Sci. U.S.A. 99, 4227-4232.

29) Hirahara, Y., Tsuda, M., Wada, Y. and Honke, K. (2000) cDNA cloning, genomic cloning, and tissuespecific regulation of mouse cerebroside sulfotransferase. Eur. J. Biochem. 267, 1909-1917. 
30) Suzuki, T., Kosaka-Suzuki, N., Pack, S., Shin, D., Yoon, J., Abdullaev, Z., Pugacheva, E., Morse, H.C. III, Loukinov, D. and Lobanenkov, V. (2010) Expression of a testis-specific form of Gal3st1 (CST), a gene essential for spermatogenesis, is regulated by the CTCF paralogous gene BORIS. Mol. Cell. Biol. 30, 2473-2484.

31) Nakajima, T., Kamijo, Y., Yuzhe, H., Kimura, T., Tanaka, N., Sugiyama, E., Nakamura, K., Kyogashima, M., Hara, A. and Aoyama, T. (2012) Peroxisome proliferator-activated receptor $\alpha$ mediates enhancement of gene expression of cerebroside sulfotransferase in several murine organs. Glycoconj. J. 2012 Oct. 13 [Epub ahead of print].

32) Bosio, A., Binczek, E. and Stoffel, W. (1996) Functional breakdown of the lipid bilayer of the myelin membrane in central and peripheral nervous system by disrupted galactocerebroside synthesis. Proc. Natl. Acad. Sci. U.S.A. 93, 13280-13285.

33) Coetzee, T., Fujita, N., Dupree, J., Shi, R., Blight, A., Suzuki, K., Suzuki, K. and Popko, B. (1996) Myelination in the absence of galactocerebroside and sulfatide: normal structure with abnormal function and regional instability. Cell 86, 209-219.

34) Fujimoto, H., Tadano-Aritomi, K., Tokumasu, A., Ito, K., Hikita, T., Suzuki, K. and Ishizuka, I. (2000) Requirement of seminolipid in spermatogenesis revealed by UDP-galactose: Ceramide galactosyltransferase-deficient mice. J. Biol. Chem. 275, 22623-22626.

35) Girault, J.A. and Peles, E. (2002) Development of nodes of Ranvier. Curr. Opin. Neurobiol. 12, 476485.

36) Chrast, R., Saher, G., Nave, K.-A. and Verheijen, M.H.G. (2011) Lipid metabolism in myelinating glial cells: lessons from human inherited disorders and mouse models. J. Lipid Res. 52, 419-434.

37) Dupree, J.L., Coetzee, T., Blight, A., Suzuki, K. and Popko, B. (1998) Myelin galactolipids are essential for proper node of Ranvier formation in the CNS. J. Neurosci. 18, 1642-1649.

38) Ishibashi, T., Dupree, J.L., Ikenaka, K., Hirahara, Y., Honke, K., Peles, E., Popko, B., Suzuki, K., Nishino, H. and Baba, H. (2002) A myelin galactolipid, sulfatide, is essential for maintenance of ion channels on myelinated axon but not essential for initial cluster formation. J. Neurosci. 22, 6507-6514.

39) Dupree, J.L., Girault, J.A. and Popko, B. (1999) Axo-glial interactions regulate the localization of axonal paranodal proteins. J. Cell Biol. 147, 11451152.

40) Takano, M., Hikishima, K., Fujiyoshi, K., Shibata, S., Yasuda, A., Konomi, T., Hayashi, A., Baba, H., Honke, K., Toyama, Y., Okano, H. and Nakamura, M. (2012) MRI characterization of spinal cord changes related to paranodal junction failure. PLoS ONE 7, e52904.

41) Marcus, J., Honigbaum, S., Shroff, S., Honke, K., Rosenbluth, J. and Dupree, J.L. (2006) Sulfatide is essential for the maintenance of CNS myelin and axon structure. Glia 53, 372-381.

42) Bansal, R., Winkler, S. and Bheddah, S. (1999) Negative regulation of oligodendrocyte differentiation by galactosphingolipids. J. Neurosci. 19, 7913-7924.

43) Bansal, R. and Pfeiffer, S.E. (1989) Reversible inhibition of oligodendrocyte progenitor differentiation by a monoclonal antibody against surface galactolipids. Proc. Natl. Acad. Sci. U.S.A. 86, 6181-6185.

44) Hirahara, Y., Bansal, R., Honke, K., Ikenaka, K. and Wada, Y. (2004) Sulfatide is a negative regulator of oligodendrocyte differentiation: development in sulfatide-null mice. Glia 45, 269-277.

45) Shroff, S.M., Pomicter, A.D., Chow, W.N., Fox, M.A., Colello, R.J., Henderson, S.C. and Dupree, J.L. (2009) Adult CST-null mice maintain an increased number of oligodendrocytes. J. Neurosci. Res. 87, 3403-3414.

46) Kajigaya, H., Tanaka, K.F., Hayashi, A., Suzuki, A., Ishibashi, T., Ikenaka, K. and Baba, H. (2011) Increased numbers of oligodendrocyte lineage cells in the optic nerves of cerebroside sulfotransferase knockout mice. Proc. Jpn. Acad., Ser. B 87, 415424.

47) Dym, M. (1983) The male reproductive system. In Histology: Cell and Tissue Biology (ed. Weiss, L.). 5th Ed., Elsevier Science, New York, pp. 10001053.

48) Jegou, B. (1993) The sertoli-germ cell communication-network in mammals. Int. Rev. Cytol. 147, $25-96$.

49) Brinster, R.L. (2002) Germline stem cell transplantation and transgenesis. Science 296, 21742176.

50) Zhang, Y., Hayashi, Y., Cheng, X., Watanabe, T., Wang, X., Taniguchi, N. and Honke, K. (2005) Testis-specific sulfoglycolipid, seminolipid, is essential for germ cell function in spermatogenesis. Glycobiology 15, 649-654.

51) Boussouar, F. and Benahmed, M. (2004) Lactate and energy metabolism in male germ cells. Trends Endocrinol. Metab. 15, 345-350.

52) Shikata, K., Suzuki, Y., Wada, J., Hirata, K., Matsuda, M., Kawashima, H., Suzuki, T., Iizuka, M., Makino, H. and Miyasaka, M. (1999) L-selectin and its ligands mediate infiltration of mononuclear cells into kidney interstitium after ureteric obstruction. J. Pathol. 188, 93-99.

53) Suzuki, Y., Toda, Y., Tamatani, T., Watanabe, T., Suzuki, T., Nakao, T., Murase, K., Kiso, M., Hasegawa, A., Tadano-Aritomi, K., Ishizuka, I. and Miyasaka, M. (1993) Sulfated glycolipids are ligands for a lymphocyte homing receptor, Lselectin (LECAM-1), Binding epitope in sulfated sugar chain. Biochem. Biophys. Res. Commun. 190, 426-434.

54) Ogawa, D., Shikata, K., Honke, K., Sato, S., Matsuda, M., Nagase, R., Tone, A., Okada, S., Usui, H., Wada, J., Miyasaka, M., Kawashima, H., Suzuki, Y., Suzuki, T., Taniguchi, N., Hirahara, Y., Tadano-Aritomi, K., Ishizuka, I., Tedder, T.F. 
and Makino, H. (2004) Cerebroside sulfotransferase deficiency ameliorates L-selectin-dependent monocyte infiltration in the kidney after ureteral obstruction. J. Biol. Chem. 279, 2085-2090.

55) Guchhait, P., Shrimpton, C.N., Honke, K., Rumbaut, R.E., Lopez, J.A. and Thiagarajan, P. (2008) Effect of an anti-sulfatide single-chain antibody probe on platelet function. Thromb. Haemost. 99, 552-557.

56) Zhou, Z., Thiagarajan, P., Udden, M., Lopez, J.A. and Guchhait, P. (2011) Erythrocyte membrane sulfatide plays a crucial role in the adhesion of sickle erythrocytes to endothelium. Thromb. Hae- most. 105, 1046-1052.

57) Takahashi, T. and Suzuki, T. (2012) Role of sulfatide in normal and pathological cells and tissues. J. Lipid Res. 53, 1437-1450.

58) Takahashi, T., Murakami, K., Nagakura, M., Kishita, H., Watanabe, S., Honke, K., Ogura, K., Tai, T., Kawasaki, K., Miyamoto, D., Hidari, K., Guo, C., Suzuki, Y. and Suzuki, T. (2008) Sulfatide is required for efficient replication of influenza A virus. J. Virol. 82, 5940-5950.

(Received Jan. 18, 2013; accepted Feb. 19, 2013)

\section{Profile}

Koichi Honke was born in 1957 and started his research career in 1984 with studies on the enzymes involved in the biosynthesis and hydrolysis of glycoconjugates at the Cancer Institute, Hokkaido University Medical School, after graduating from the Hokkaido University School of Medicine. He worked at the Osaka Medical Center for Maternal and Child Health as chief researcher from 1995 to 1999 and the Department of Biochemistry, Osaka University Medical School as associate professor from 1999 to 2003. He was then promoted to full professor of the Kochi Medical School in 2003. His major contribution in the field of glycobiology was purification, molecular cloning and gene targeting of a sulfotransferase responsible for the biosynthesis of sulfatides and discovery of the $\beta \mathrm{Gal} 3$ - $O$-sulfotransferase gene family. Now his research focus shifted to the

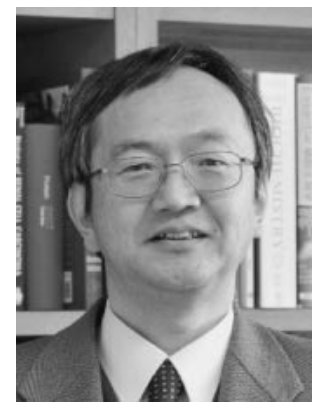
biogenesis of the membrane microdomains with establishment of a novel labeling method called EMARS for identification of cell surface molecular clustering in living cells. 